\title{
Light Microscopy Analysis of Bone Response to Implant Surfaces
}

\section{Jae-Hyuk Sim and In-Sung Yeo*}

Department of Prosthodontics, School of Dentistry and Dental Research Institute, Seoul National University, 101 Daehak-ro, Jongno-gu, Seoul 03080, Korea

*pros53@snu.ac.kr

Abstract: Light microscopy histomorphometry is a useful tool in dentistry to evaluate early biologic response at boneimplant interfaces. Using this analysis method, a quantitative study compared the bone response to two types of implant surface. Implants were inserted in the tibia of two New Zealand white rabbits. After sacrifice, the tibial bone blocks were sectioned and processed in the undemineralized state. Two histomorphometric quantities were measured: boneto-implant contact and bone area. Similar bone response was found for each implant surface. While this microscopy technique is limited to two dimensions, it was adequate for estimating bone response to implant surfaces.

\section{Introduction}

Clinically, dental implants are excellent replacements for missing teeth. Originally, implants were made of commercially pure titanium (cp Ti) that required a long time for biological attachment to the bone, also known as osseointegration. The surfaces of implants have been modified to accelerate biological bone response to it, resulting in the decrease of a patient's edentulous period, which is extremely significant in a clinical context $[1,2]$. The concept of osseointegration has been defined at the light microscopy level [3]. Histomorphometry in an in-vivo study has therefore been recognized as one of the most useful methods for the evaluation of early biologic response at the interface between the bone and the implant surface [4]. Two parameters in histomorphometric analysis are widely used to compare early bone responses to different implant surfaces: (1) bone-toimplant contact (BIC), defined as the ratio of the bone length in contact with the implant surface to the total length of the surface in a light microscopy image and (2) bone area (BA), defined as the ratio of the bone formed between the implant threads to the total area between the threads.

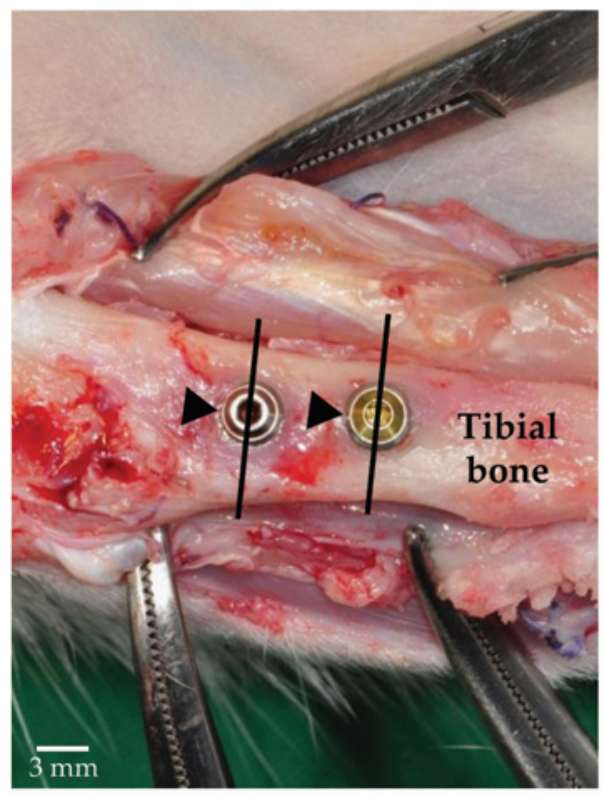

(a)

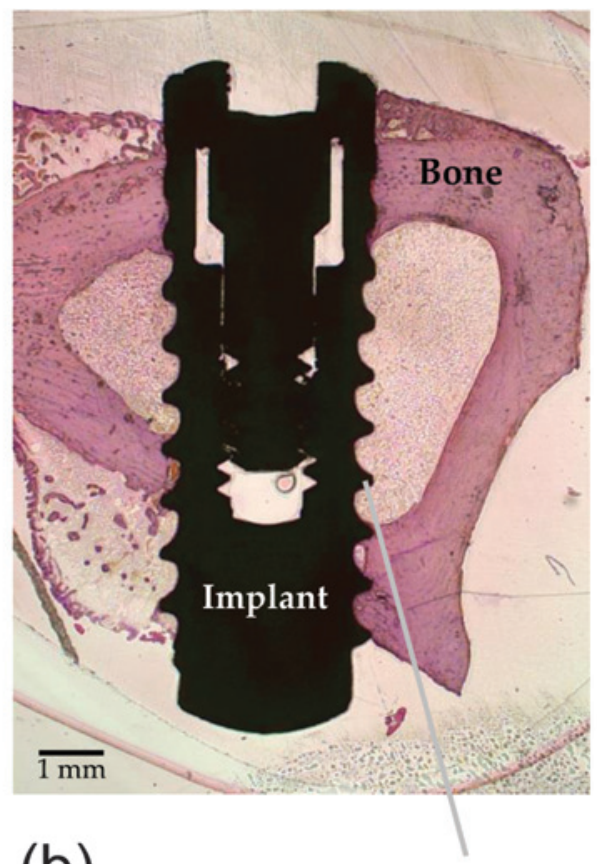

(b)

\section{Implant} thread



(c)

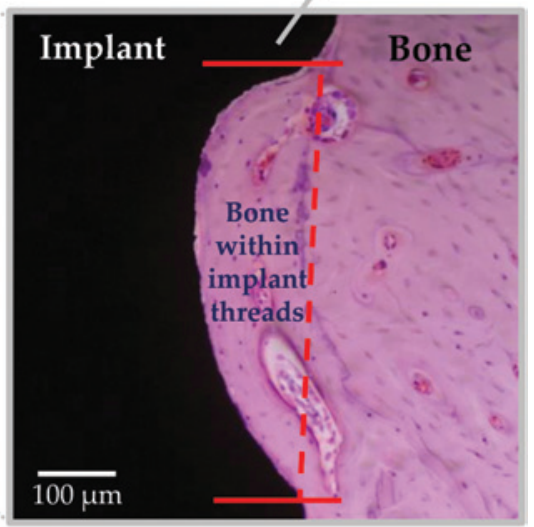

(d)

Figure 1: The implants (black arrowheads) were inserted into the rabbit tibiae in this study (a). The black lines in this figure (a) indicate the cross-sectional lines to prepare the specimens for light microscopy analysis. The real image of a whole specimen is shown (b), and its schematic diagram is also presented (c). The implants were $3.3 \mathrm{~mm}$ in diameter and $10.0 \mathrm{~mm}$ in length. Notice the gap between the implant and the upper part of the cortical bone. The implant was anchored to the lower part of the cortical bone. The interface (gray square) in (c) between the implant and the bone was the main area for the histomorphometric analysis in this study. The real enlarged image of this area is also shown (d). 


\section{N口 ᄃ口MPA口MISE ATDMIL RESDLUTIDN + FAST ANALYSIS}

The New "GO-To" TEM from JEOL

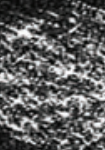

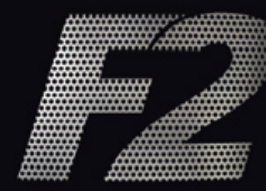

- Cold FEG boosts probe current

- Fast 3D EDS with dual SDD

- Wide-field STEM-EELS spectrum imaging

- Push-button beam conditions

- Ultra-stable, ultra-fast

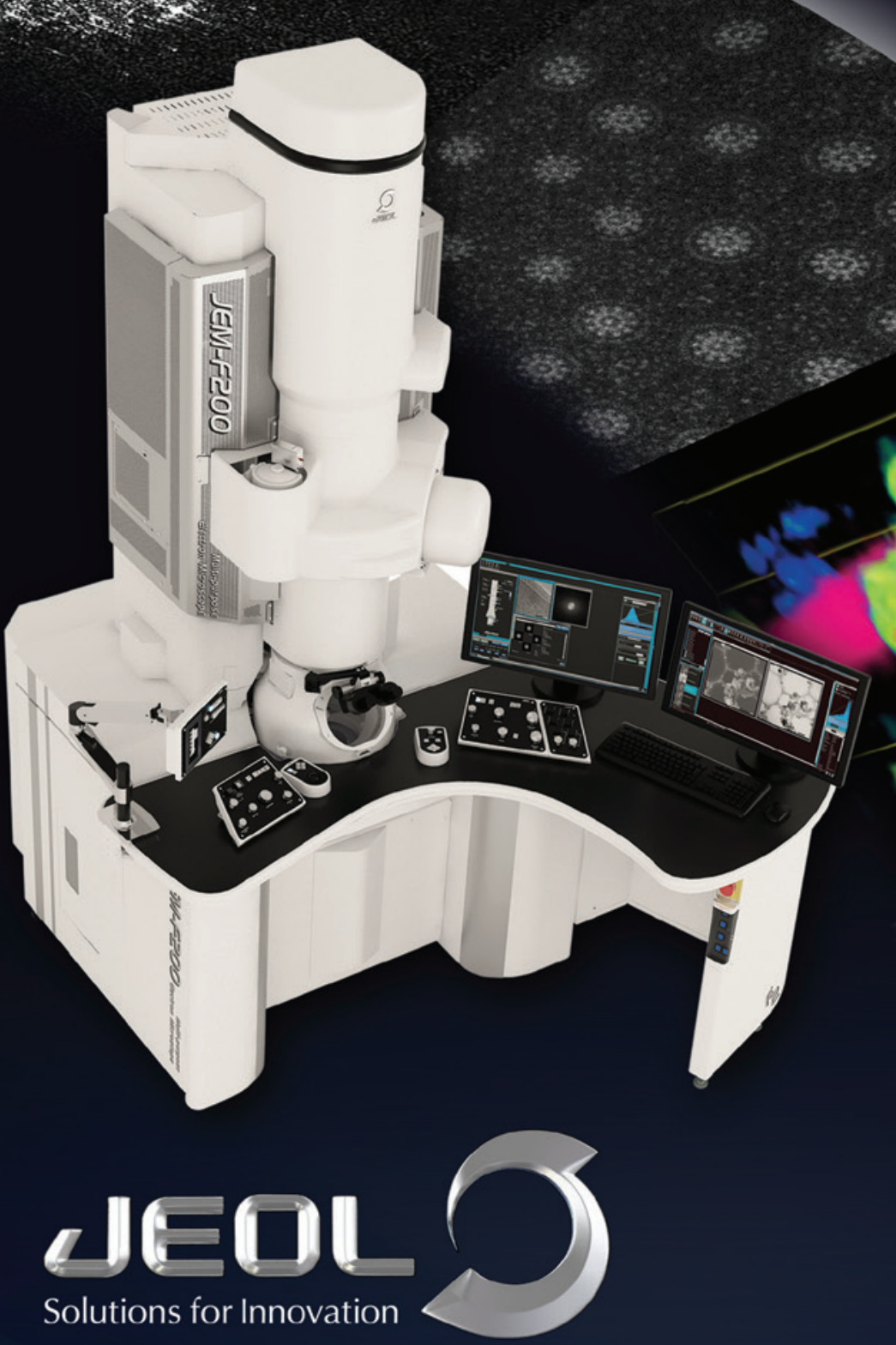


Previous studies have shown that the modified sandblasted, large grit and acid-etched (modSLA) surface has a significantly superior bone response to its predecessor, the traditional hydrophobic sandblasted, large grit and acid-etched (SLA) one, both in vitro and in vivo $[5,6]$. However, other studies have reported no significant differences between the modified surfaces [7-12]. These contradictory results can be explained by differences in the techniques used for animal sacrifice and the type of animal used. A previous animal experiment using dogs showed similar BIC and biomechanical results between the modSLA and anodized Ti surfaces [7]. Another study comparing calcium phosphate-coated, anodized, and sandblasted surfaces found no significant differences among them with respect to the same histomorphometric parameters used in the present study [11]. Quantitative comparison of BIC and BA showed no significant differences between calcium phosphate- and hydroxyapatite-coated surfaces; modSLA and fluoride-treated surfaces; fluoride-treated and anodized surfaces; and calcium phosphate-coated anodized Ti, SLA, and anodized implant surfaces [8-10,12]. In general, when the modified surfaces were compared

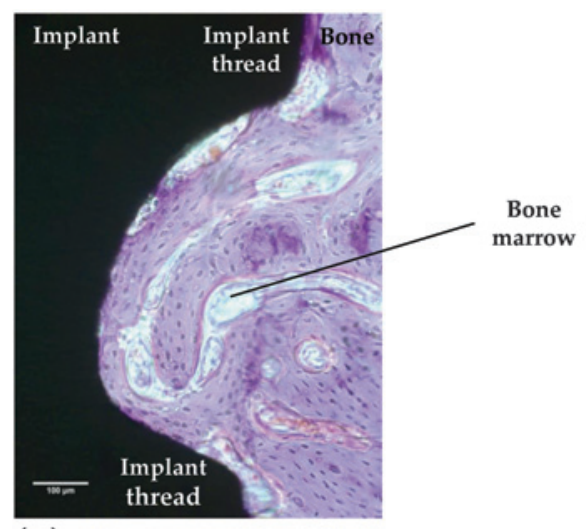

(a)



(c)

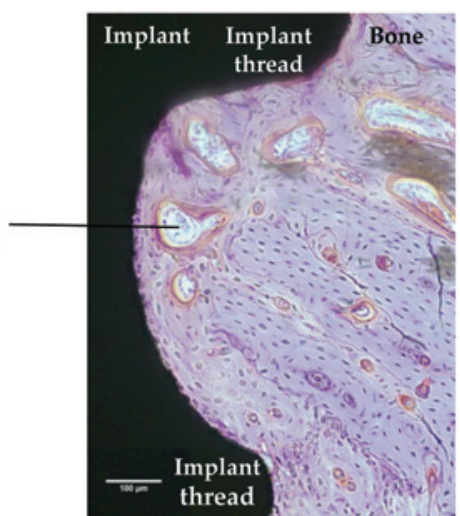

(b)



(d)

Figure 2: The histological images ( $a$ and b) and the measurements of histomorphometric quantities (c and d) are shown in these light microscopy images. For clearer understanding, the schematic diagrams are also presented. The parts of the implant that the bone attaches to are designated as 1 (red lines), and those without bone contact are as 2 (green lines) (c). BIC is calculated using the following formula: (the sum of $1 \mathrm{~s}$ ) / (the sum of $1 \mathrm{~s}$ and $2 \mathrm{~s}$ ). The total area between the implant threads is designated as 1 (red line polygon), and the areas without bone are designated as 2 (green line polygons) (d). BA is calculated using the following formula: [(the area of 1) - (the sum of 2s)] / (the area of 1). Notice the limitation of these image analyses: they are two-dimensional analyses for a three-dimensional object.

in vivo with the cp Ti surface without

any further surface modification, the histomorphometric results clearly exhibited superior early bone response in the modified surfaces $[2,11]$. However, light microscopy analysis, including BIC and $\mathrm{BA}$, found few comparative advantages in biocompatibility or in bone formation when the modified surfaces are compared with each other, especially when other modified surfaces are compared with the traditional SLA surface $[9,10]$.

This article describes the use of the BIC and BA parameters in an investigation of early bone responses to modified implant surfaces. The results lead to a consideration of the limitations of light microscopy analysis.

\section{Materials and Methods}

Implant surfaces. Eight screw-shaped implants $(3.3 \mathrm{~mm}$ in major diameter and $10 \mathrm{~mm}$ in length) were prepared from grade IV cp Ti. Four implants had modSLA surfaces (SLActive, Institute Straumann AG, Basel, Switzerland), wherein they were first sandblasted using large grit, acid-etched, and then chemically modified to have hydrophilic properties [13]. The surfaces of the remaining four implants were SLA, sandblasted with large grit and acid-etched only (Deep Implant System, Seoul, Korea) [13]. The overall images of the investigated surfaces were obtained using a scanning electron microscope (Hitachi S-4700 FE-SEM).
Implant insertion. In-vivo surgery was performed on rabbits with the approval of the Animal Research Committee of Seoul National University and in accordance with the guidelines provided by the Institute of Laboratory Animal Resources at Seoul National University. The surgery protocol has been described in detail previously [9, 11]. This study used two female New Zealand white rabbits that were aged approximately 1 year. Their weights ranged from 2.0 to $2.5 \mathrm{~kg}$. The rabbits received intravenous anesthesia with tiletamine/ zolazepam 15 mg/kg (Zoletil 50, Verbac Korea Co. Ltd., Seoul, Korea) and xylazine $5 \mathrm{mg} / \mathrm{kg}$ (Rompun, Bayer Korea Ltd., Seoul, Korea). The shaved skin in the proximal tibial area was decontaminated with betadine, and a preoperative antibiotic (Cefazolin, Yuhan Co., Ltd., Seoul, Korea) was intravenously administered before surgery. Incision, muscle dissection, and periosteal elevation were performed to expose the tibial bone. The medial and distal surfaces of the proximal tibia were first drilled with a $2.0 \mathrm{~mm}$ diameter round burr for implant installation, and the drilled hole was then successively enlarged bicortically to $3.0 \mathrm{~mm}$ diameter. The hole on the medial surface was enlarged to a greater extent $(3.5 \mathrm{~mm}$ diameter $)$ to produce a gap between the implant and the bone (Figure 1). Each rabbit received four implants (two implants in each tibia) using the $2 \times 2$ Latin 

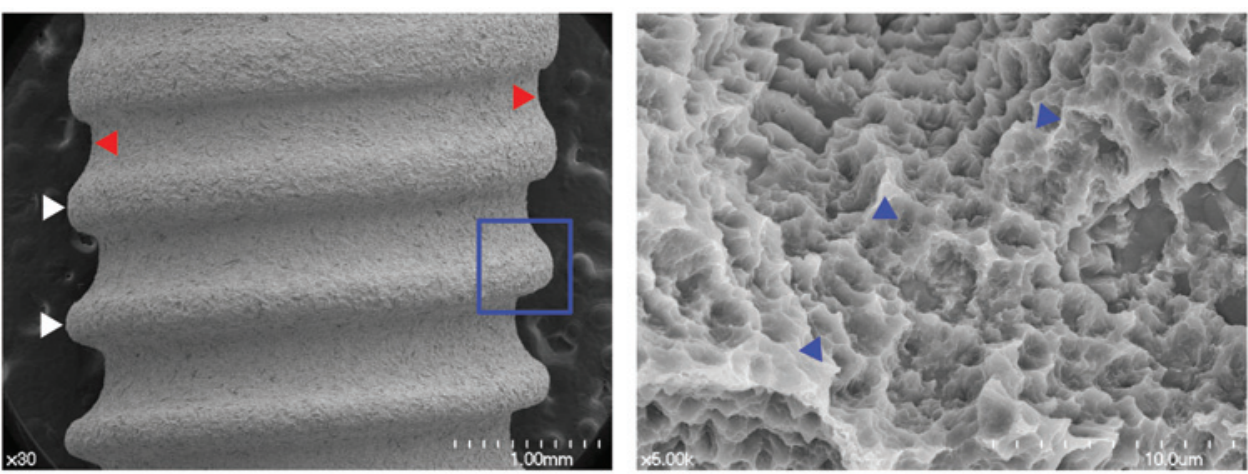

(a) (b)

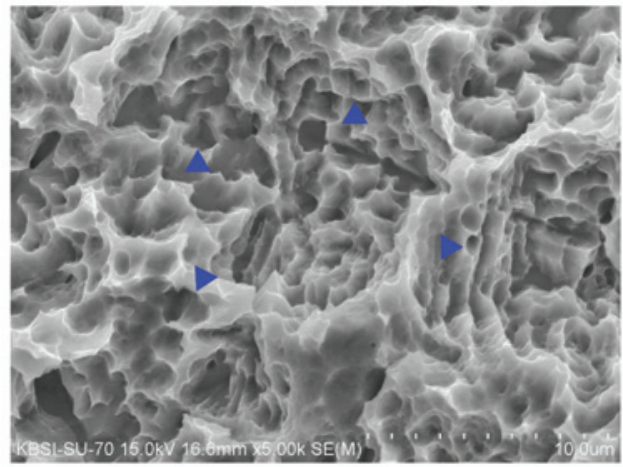

(c)

Figure 3: SEM images of the implants. The overall shape of the implants used in this study is shown (a). The implant threads (white arrowheads) and thread valleys (red arrowheads) are observed in this low-magnified image. The higher-magnified images are obtained from the blue square area for each implant. Note that the similar irregular, honeycomb-shaped structures (blue arrowheads) are found in both the modSLA (b) and SLA (c) implants. Such sharp-edged, honeycomb-like irregularities are considered to be produced from the blasting and etching procedures.

square randomization method. The surgical sites were sutured in layers after implant placement.

Light microscopy and image analysis. The rabbits were kept in separate cages. Four weeks after surgery, they were anesthetized and sacrificed by the intravenous injection of potassium chloride. The implants were surgically removed en bloc with the adjacent collars of bone and immediately fixed in $10 \%$ neutral formaldehyde. Specimen preparation for light microscopy in this case has been described previously [14]. Briefly, the un-decalcified specimens were embedded in resin (Technovit 7200 VLC, Kultzer, Wehrheim, Germany) and then ground using the Exakt system (Exakt Apparatebau, Norderstedt, Germany) to an approximate thickness of $50 \mu \mathrm{m}$. Next, they were stained with hematoxylin and eosin and examined under a light microscope (Olympus model BX). The $\mathrm{BIC}$ and $\mathrm{BA}$ ratios were calculated using image analysis software (Kappa PS30C ImageBase, Kappa Opto-electronics $\mathrm{GmbH}$, Gleichen, Germany) connected to the microscope (Figure 2).

Table 1: Mean and SD of $B I C$ and $B A$ at 4 weeks after implant insertion.

\begin{tabular}{|l|l|l|}
\hline & BIC (\%) & BA (\%) \\
\hline modSLA & $75.4 \pm 16.3$ & $94.0 \pm 7.3$ \\
\hline SLA & $83.0 \pm 8.7$ & $91.5 \pm 2.7$ \\
\hline P-value & 0.465 & 0.715 \\
\hline
\end{tabular}

BIC: bone-to-implant contact; BA: bone area
A Wilcoxon signed rank test was carried out to find significant differences in histomorphometry between the investigated surfaces. The level of significance was set at $5 \%$.

\section{Results}

Figure 3 shows SEM images of the test and the control surfaces. Both surfaces displayed honeycomb-shaped irregularities that were similar to each other and were a result of the grit blasting and acid etching procedures.

The mean and standard deviations (SDs) of the BIC ratio and BA are reported in Table 1 for the modified SLA (modSLA) and the SLA surfaces. The histomorphometric analysis revealed no statistically significant differences (Figure 4) between two surfaces. No significant differences in BIC were observed between the modSLA and SLA surfaces $(p>0.05)$, although the SLA implant showed a higher mean value. Moreover, there were no significant differences in BA between the groups $(p>0.05)$.

\section{Discussion}

Rough Ti surfaces are known to affect bone responses. However, the exact reactions to the roughened surfaces have been little understood at the cellular or the molecular level $[2,6,15]$. It is unknown which surface topographic morphology has more affinity to bone forming cells when the surfaces have similar roughness $[2,15]$. For hydrophilicity, there appears to be no consensus concerning the mechanism having positive effects on bone healing, although the higher expression of osteogenic markers and superior in-vivo biologic responses were reported in some previous studies [5, 6]. High affinity of blood and water shell surrounding biomolecules (such as proteins) to the hydrophilic surface is considered as a cause [5]. However, such an effect is expected to be almost negligible, which is why this study excluded this effect, and the results were similar between the modSLA and SLA surfaces. Further studies of the biological mechanisms on the roughened or hydrophilic surface are required.

Although light microscopy histomorphometry is a widely accepted tool for the evaluation of early biological response at the bone-implant interface, it appears to have some limitations that should be taken into consideration. Firstly, light microscopy examination allows only two-dimensional measurement because the specimen is prepared by vertically or horizontally crosssectioning a three-dimensional volumetric object. However, a single cross-sectional view may not adequately represent the whole three-dimensional image of bone response around the implant surface. Moreover, the selection of a location for sectioning and the resultant histological views are both probabilistic to an extent. Recently, efforts to overcome this limitation have been made. The three-dimensional extensions for BIC and $\mathrm{BA}$ are BIC area and bone volume, respectively. Recently, both 
the BIC area and bone volume have been calculated using microcomputed tomography $[16,17]$.

Secondly, histomorphometry has no biomechanical point of view, which is important for implants that are inserted into the human body. For example, BIC does not consider the quality of binding between the bone and implant, and every contact of the bone to the implant surface is assumed to be of the same quality. Therefore, osseointegration is considered to improve as BIC increases. Various forces are applied on implants inserted into the human body, with dental implants receiving compressive and shearing forces when the patient chews food. Therefore, the biomechanical aspects at the interface between the implant and biological surfaces should be investigated. Hence, it is important to understand that histomorphometric analysis should be supported by biomechanical data. Removal torque is used for the biomechanical back-up, which is defined as minimal torque to break osseointegration, or bonding between the bone and screw-shaped implant, when a counterclockwise torque is applied to the osseointegrated, righthanded screw implant [11]. However, the removal torque value has the limitation that screw shape and implant morphology also affect the value, as well as the implant surface. Recently, other biomechanical data, including angular momentum of a rotating body, are being analyzed for evaluating the osseointegration quality of the surface [18].

\section{Conclusion}

The results of this in-vivo, light microscopy histomorphometric analysis showed that hydrophilic modSLA Ti surfaces exhibited no significant superiority in bone response compared with the traditional hydrophobic SLA Ti surfaces, even though previous studies demonstrated improved rate and quantity of bone formation around the modified implant surfaces than the cp Ti ones. Light microscopy analysis is widely used and is considered to be an adequate tool for the evaluation of early bone response to the implant surface. However, histomorphometric examination in implantology is limited by the fact that it allows only two-dimensional analysis, at present. Further investigations are required to extend this to three-dimensional analysis.

\section{References}

[1] A Wennerberg and T Albrektsson, Int J Oral Max Impl 25(1) (2010) 63-74.

[2] IS Yeo, Open Biomed Eng J 8 (2014) 114-49.

[3] P-I Branemark et al., Tissue-integrated prostheses: osseointegration in clinical dentistry, Quintessence Publishing, Kempten, 1985, 11-13.

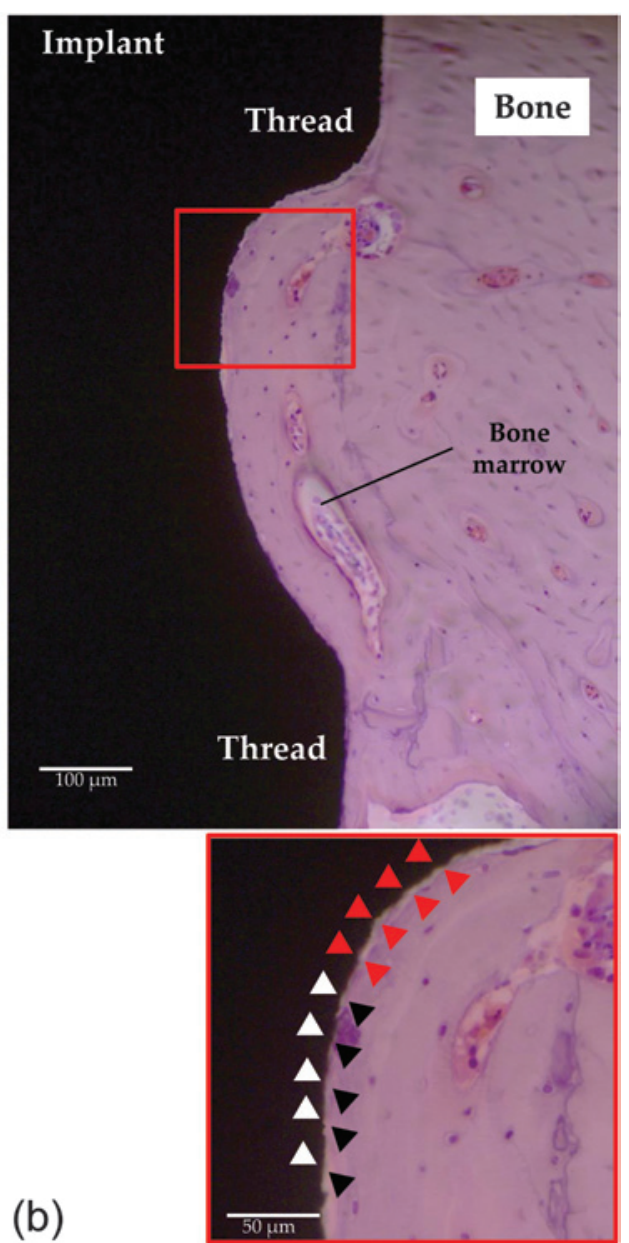

(b)

Figure 4: In the light microscopy views, the modSLA (a) and SLA (b) implants show spaces between the threads that are filled with bone. No noticeable differences in bone responses to the surfaces were observed in this study. The direct contact of the bone to the implant surface (black and white arrowheads) is shown in the higher-magnified images (red squares), while a thin void (red arrowheads) is sometimes observed on the images of both the surfaces. Such a void is considered not to be the direct contact, or the bone is not considered to be integrated to the implant surface.

[4] PG Coelho et al., J Biomed Mater Res B Appl Biomater 88(2) (2009) 579-96.

[5] MM Bornstein et al., Clin Oral Implants Res 19(3) (2008) 233-41.

[6] I Wall et al., Bone 45(1) (2009) 17-26.

[7] EA Bonfante et al., Clin Oral Implants Res 24(12) (2013) 1375-80.

[8] JY Choi et al., Implant Dent 21(2) (2012) 124-28.

[9] YS Hong et al., Implant Dent 23(5) (2014) 529-33.

[10] JW Koh et al., Int J Oral Max Impl 28(3) (2013) 790-97.

[11] IS Yeo et al., J Biomed Mater Res B Appl Biomater 87(2) (2008) 303-11.

[12] IS Yeo et al., J Korean Phys Soc 57(6) (2010) 1717-20.

[13] F Rupp et al., J Biomed Mater Res A 76(2) (2006) 323-34.

[14] K Donath and G Breuner, J Oral Pathol 11(4) (1982) 318-26.

[15] LF Cooper, J Prosthet Dent 84(5) (2000) 522-34.

[16] SW Pyo et al., Int J Oral Max Impl 29(2) (2014) 478-84.

[17] K de Faria Vasconcelos et al., Clin Oral Implants Res (2016), doi: 10.1111/clr.12799.

[18] TK Kwon et al., Implant Dent 21(5) (2012) 427-32. 


\section{İTESCAN}

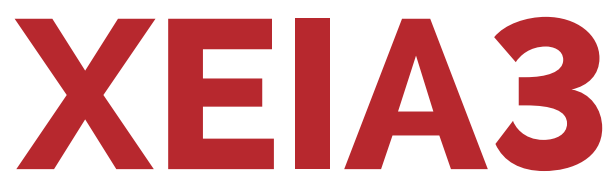

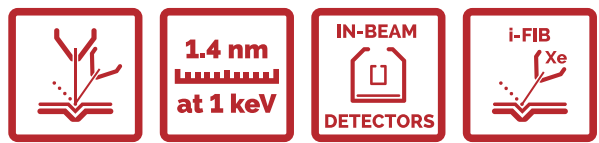

Plasma FIB

Extending the horizons of FIB-SEM applications

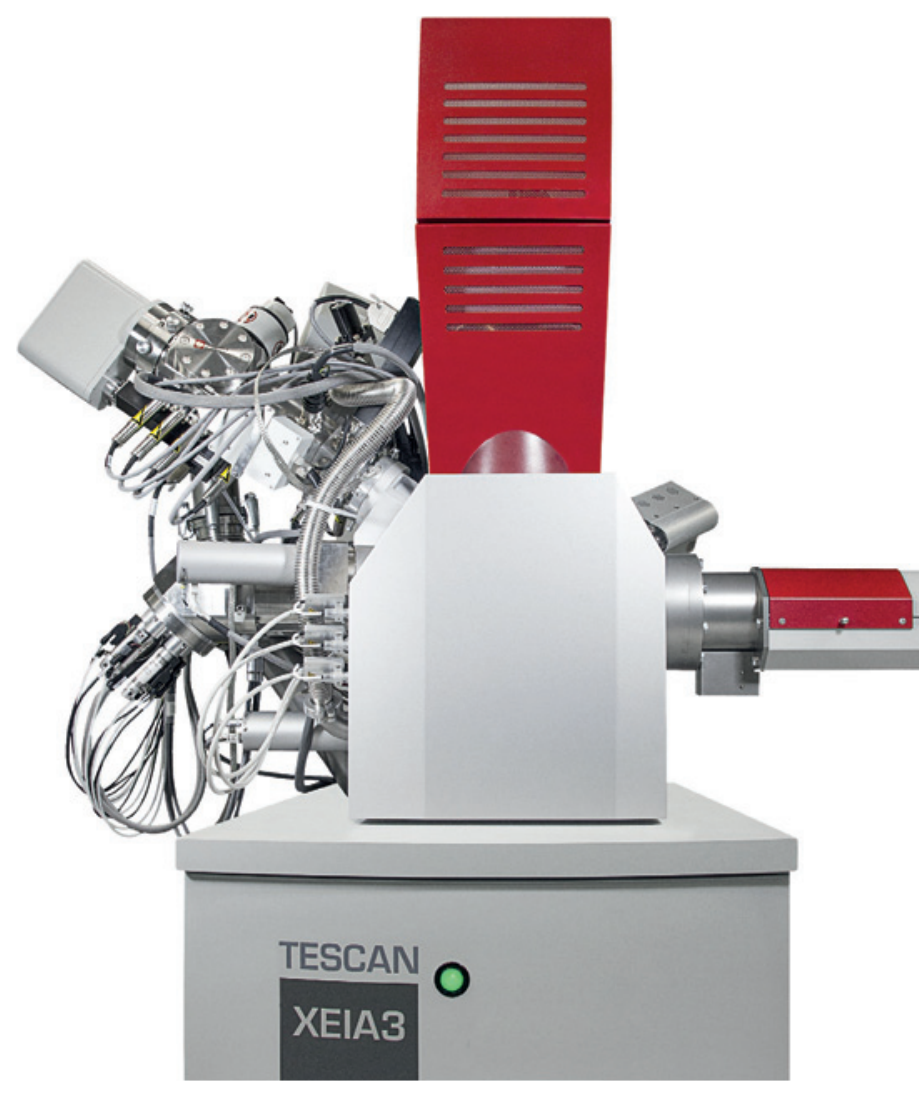

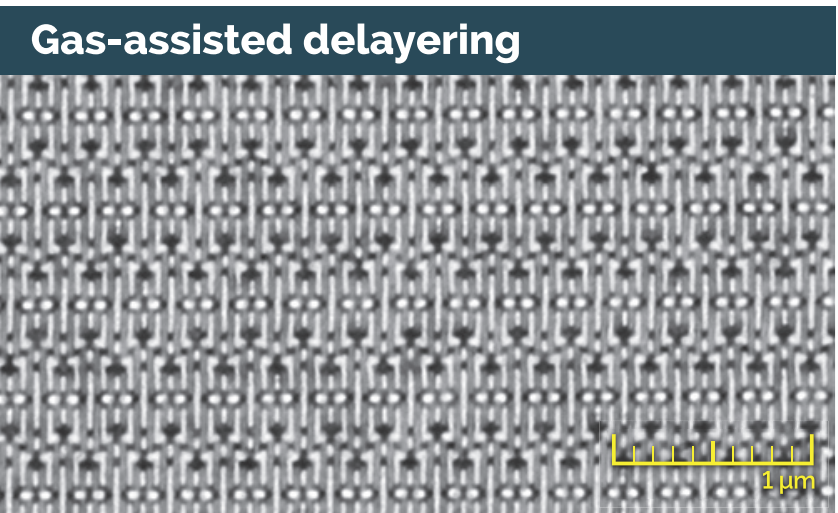

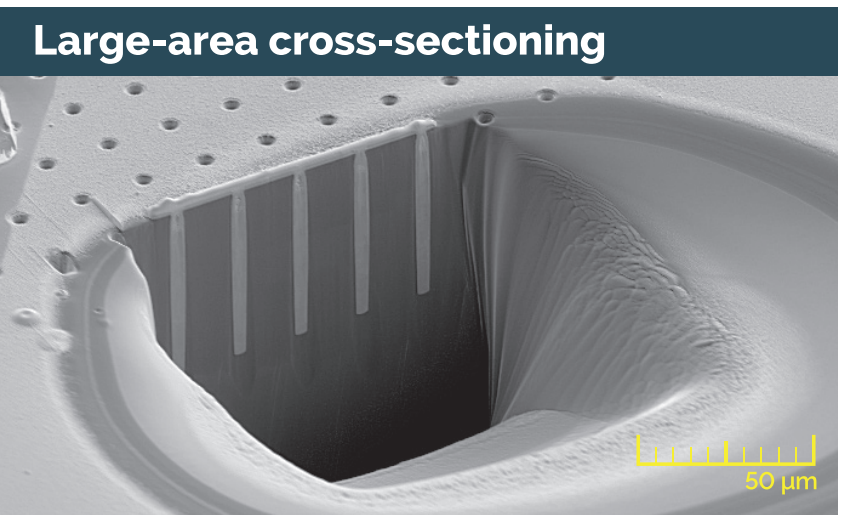

Large volume FIB-SEM tomography
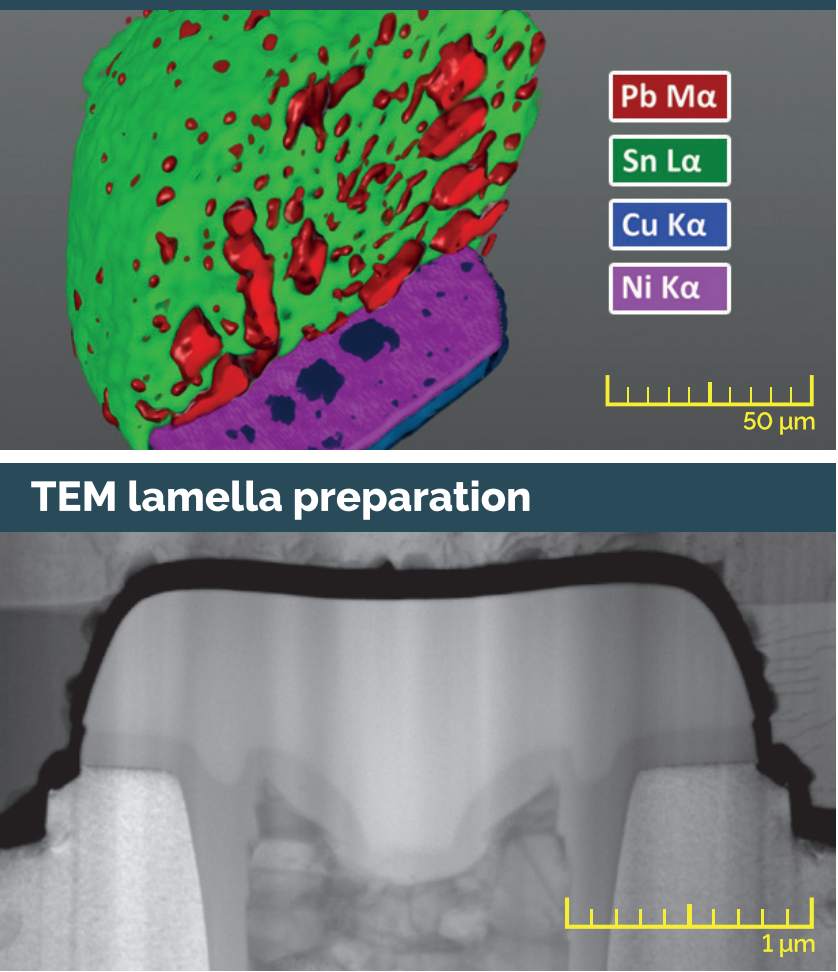

\section{TESCAN USA Inc.}

765 Commonwealth Drive I Suite 101 Warrendale, PA 15086 wwww.tescan-usa.com 